\title{
ESCUELA Y DEMOCRACIA. EXPERIENCIAS EN FORMACIÓN CIUDADANA EN ESTABLECIMIENTOS EDUCACIONALES DE LA REGIÓN METROPOLITANA
}

\author{
Rodrigo Escobar San Martín ${ }^{1}$
}

\section{RESUMEN/ ABSTRACT}

El artículo presenta el trabajo realizado en la asesoría en planes de formación ciudadana a distintos establecimientos educacionales municipales del Departamento Provincial Sur de la Región Metropolitana. La asesoría tiene como propósito apoyar la implementación de la Ley 20.911 destinada a fortalecer la formación ciudadana en los establecimientos educacionales reconocidos por el Estado. En primer lugar, se hace una revisión de la literatura especializada sobre formación ciudadana y escuela democrática, con principal énfasis en los documentos de instituciones globales que se preocupan de este tema. Se consideran también los objetivos y alcances de la ley, además de las políticas educativas nacionales. A continuación, se relata el trabajo realizado en conjunto con los establecimientos educacionales del Departamento Provincial Sur y los resultados de esta experiencia de asesoramiento educativo. Finalmente, se plantean algunas reflexiones sobre la experiencia, en particular sobre las tensiones que se producen entre los objetivos de la ley y las culturas escolares, tanto en cuestiones de forma (¿cómo abordar la formación ciudadana?), como de fondo (¿cómo articular la ley con los documentos institucionales?).

Palabras claves: Escuela democrática; formación ciudadana; planes de formación ciudadana; cultura escolar.

The article presents the work carried out in the advice on Citizenship Training Plans to public schools of the Southern Provincial Department of the Metropolitan Region (Chile). The purpose of the counseling is to support the implementation of Law 20.911, aimed at strengthening citizenship training in public schools. First of all, a review of the specialized literature on citizenship education and democratic school is made, with main emphasis on the documents of global institutions that are concerned with this topic. The objectives and scope of the law are also considered, in addition to the national educational policies

\footnotetext{
${ }^{1}$ Profesional del proyecto "Construcción de ciudadanía en el sistema escolar", de la Universidad Católica Silva Henríquez; Profesor de Filosofía; rodrigo_escobarsm@hotmail.com
} 
The following is a report of the work carried out in conjunction with the educational establishments of the Southern Provincial Department of the Metropolitan Region (Chile) and the results of this educational counseling experience. Finally, there are some reflections on experiences that stress the objectives of the law with school cultures, in terms of how to address civic education and how to articulate the law in institutional documents.

Keywords: Democratic school; citizenship education; citizenship training plans; school culture.

\section{INTRODUCCIÓN}

No cabe duda que, hoy en día, uno de los temas más importantes de la agenda educativa es la formación ciudadana. Desde los inicios del presente siglo la formación ciudadana ha sido un tema recurrente, objeto de debates y de comisiones que progresivamente han ido situando en la agenda política la necesidad de educar en ciudadanía. La crisis política, expresada en actos de corrupción y en la preocupante merma en la participación política en los últimos años, hace más necesario que nunca educar en ciudadanía, ya no solo como parte de un proceso formativo, sino que principalmente para fortalecer la convivencia democrática y la democracia misma.

Los informes del Programa de las Naciones Unidas para el Desarrollo (PNUD, 2002; 2004; 2014; 2015; 2017) señalan la importancia de la ciudadanía para la consolidación de la democracia. Una ciudadanía empoderada y participativa implica, a su vez, una democracia saludable. Es por ello que, en los distintos informes elaborados en relación a la política en nuestro país, se enfatiza la necesidad de tratar los temas contingentes en materia política dentro de un proceso formativo, señalando a la escuela como promotora de ciudadanos.

Como señala Touraine (2015), el desarrollo cultural de una sociedad invita a relacionarnos en democracia, a convivir democráticamente, especialmente en Latinoamérica, donde, tal como plantea Norbert Lechner (1987; 1988), la convivencia político-social ha sufrido los embates del autoritarismo, teniendo como consecuencia democracias frágiles, gracias a la persistencia de irrupciones traumáticas del pasado o a la herencia política autoritaria que aún sobrevive en las incipientes democracias de la región. 
En el marco de estas consideraciones, a continuación se presenta el trabajo realizado en la asesoría en planes de formación ciudadana a distintos establecimientos educacionales municipales del Departamento Provincial Sur de la Región Metropolitana. Esta asesoría tuvo como propósito apoyar la implementación de la Ley 20.911, destinada a fortalecer la formación ciudadana en los establecimientos educacionales reconocidos por el Estado.

\section{MARCO TEÓRICO}

La política pública en formación ciudadana que se materializó en la Ley 20.911 tiene como antecedente inmediato las propuestas del Consejo Asesor Presidencial contra los conflictos de interés, el tráfico de influencias y la corrupción (2015) que enfatizan la necesidad de "un sistema educacional que forme en valores cívicos de respeto a la convivencia y fomento del bienestar común" (p. 89). Este Consejo propuso incluir la formación ciudadana en las bases curriculares desde el segundo nivel de transición hasta el cuarto año de educación media; incluir la formación ética y ciudadana en los proyectos educativos institucionales de los establecimientos educativos; e incluir en la formación inicial docente la formación ciudadana, ética y el pensamiento crítico.

También habría que considerar como un antecedente los cambios políticos de transformación y modernización de la educación que a partir de los años 90, en pleno proceso de retorno a la democracia en Chile, fueron impulsados por los gobiernos de la Concertación. La preocupación por la formación ciudadana comienza el año 1994, año en el que se esbozan los lineamientos básicos para abordar los elementos políticos y ciudadanos que deben ser parte de los fines de la educación. Asimismo, con la creación de la Comisión Nacional de Modernización de la Educación comienza a instalarse el discurso de avanzar en la calidad del sistema educativo. A raíz del informe emanado de esta comisión (1994) se plantea como una de las primeras medidas de la reforma educacional la eliminación de la asignatura de educación cívica del currículum nacional en la escuela junto con su transversalización. A partir del año 1998 la formación política y ciudadana deja de ser algo propio de una asignatura en particular para pasar a ser un elemento transversal 
dentro del currículo escolar, es decir, se pasa de una concepción minimalista a la hora de abordar la educación cívica hacia una concepción maximalista.

Un estudio del año 2004 señala que los contenidos asociados al área de formación ciudadana son impartidos principalmente por tres asignaturas: Historia y Geografía, Lenguaje y Comunicación, y Filosofía. Los aprendizajes en ciudadanía se fomentan también en otros espacios de formación educativa como lo es Orientación y Consejo de Curso. El problema que surge es que todas estas asignaturas y actividades que tienen componentes relacionados con la formación ciudadana no se articulan entre sí. No se estaría llevando a cabo, por tanto, aquel supuesto de la concepción maximalista que establece la transversalidad del currículum, aunque existan dentro de los objetivos fundamentales transversales elementos guías de trabajo en esta materia dirigidos hacia los/as docentes y directivos (Cerda, Egaña, Magendzo, Santa Cruz \& Varas, 2004).

También en el año 2004 se crea la Comisión de Formación Ciudadana, la cual presenta un informe donde se señala la necesidad de considerar esta materia como una instancia educativa formativa, en que tanto el currículum, las prácticas en el aula y los proyectos educativos se orienten hacia una reconfiguración de la institucionalidad educativa en virtud de los acontecimientos políticos acaecidos a inicio de siglo. Del mismo modo, se hace énfasis en las tendencias educativas globales propias del siglo XXI, en las transformaciones político-culturales y en el florecimiento un tanto tímido del primer movimiento estudiantil del año 2001-2002 que fue conocido como "mochilazo".

Este primer movimiento estudiantil fue la antesala de las grandes movilizaciones del año 2006. Este segundo movimiento estudiantil tuvo como triunfo simbólico la derogación de la Ley Orgánica Constitucional de Enseñanza (LOCE) que fue reemplazada por la promulgación de la Ley General de Educación (LGE) que, en líneas generales, no introdujo modificaciones significativas en materia de formación ciudadana, aun cuando su necesidad había sido planteada en el año 2004 por la Comisión de Formación Ciudadana. Sin embargo, el Gobierno de la época avanza en una propuesta ya instituida en el año 1994 por la Comisión Nacional de Modernización de la Educación que instala como elemento fundamental mejorar la calidad del sistema educativo. 
En el año 2009, el Ministerio de Educación de Chile (Mineduc) participa en un estudio internacional de educación cívica y formación ciudadana. Los resultados de dicho estudio fueron entregados en 2010, evidenciando una fragilidad en la formación ciudadana $\mathrm{y}$, por tanto, la necesidad de buscar estrategias para lograr mejores resultados en este tipo de prueba de carácter estandarizado a nivel mundial (Mineduc, 2003; 2013; ACE, 2016). De esta manera, el Gobierno propone avanzar en la obligatoriedad de un plan de formación ciudadana para cada uno de los establecimientos educativos del país. En primer lugar, se propone una asignatura de formación ciudadana y al mismo tiempo acciones concretas tanto institucionales como en la transversalidad del currículum que aborden el tema de la formación ciudadana en su conjunto, es decir, se asume que el concepto de una cultura democrática y ciudadana debe ser uno de los fundamentos principales de una acción educativa en que tanto la sociedad como la escuela dan vida a la democracia real (Apple \& Beane, 1999).

A continuación se presenta una cronología con los principales hitos en materia educativa que afectan directamente a la formación ciudadana una vez recuperada la democracia en Chile:

\begin{tabular}{|c|c|}
\hline $\begin{array}{l}\text { 1994: Comisión Nacional de Modernización } \\
\text { de la Educación. }\end{array}$ & $\begin{array}{l}\text { 2009: Estudio Internacional de Educación } \\
\text { Cívica y Formación Ciudadana. }\end{array}$ \\
\hline $\begin{array}{l}\text { 1995-96: Transversalización en la Reforma } \\
\text { Educacional. }\end{array}$ & 2009: Ley General de Educación. \\
\hline $\begin{array}{l}\text { 1998: Eliminación de la asignatura de } \\
\text { educación cívica del currículum nacional. }\end{array}$ & 2011: Ley de Aseguramiento de la Calidad. \\
\hline $\begin{array}{l}\text { 1999: Estudio Internacional de Educación } \\
\text { Cívica y Formación Ciudadana. }\end{array}$ & 2015: Ley de Inclusión. \\
\hline 2002: Política de Convivencia Escolar. & $\begin{array}{l}\text { 2015: Consejo Asesor Presidencial contra } \\
\text { los conflictos de interés, el tráfico de } \\
\text { influencias y la corrupción. }\end{array}$ \\
\hline $\begin{array}{l}\text { 2004: Estudio de Formación Ciudadana y } \\
\text { Prácticas Docentes. }\end{array}$ & 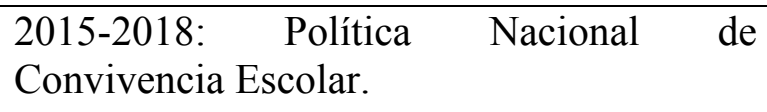 \\
\hline 2004: Comisión de Formación Ciudadana. & $\begin{array}{l}\text { 2016: Ley } 20.911 \text { crea Plan de Formación } \\
\text { Ciudadana. }\end{array}$ \\
\hline
\end{tabular}




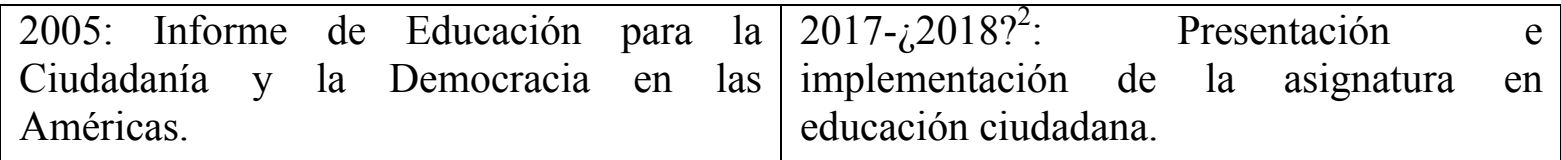

Cronología de estudios y políticas asociadas a formación ciudadana. Fuente: elaboración propia.

Los fundamentos que plantea el Mineduc para entender a qué se hace referencia con el concepto de formación ciudadana son extraídos, principalmente, del IV Seminario de Ministros de Educación que se llevó a cabo en Trinidad y Tobago y también de la obra de los autores Cristián Cox, Rosario Jaramillo y Fernando Reimers, titulada Educar para la Ciudadanía y la Democracia en las Américas: una Agenda para la Acción (2005). Además, el Mineduc considera los criterios asociados a la Prueba Internacional de Conocimientos Ciudadanos, más conocida como ICCS por sus siglas en inglés (Schulz, Fraillon, Ainley, Losito, \& Kerr, 2008) $)^{3}$.

A este respecto se plantea que, a nivel latinoamericano, el tránsito de la educación cívica a la Formación Ciudadana implica una ampliación de concepto y de sentido en tres áreas fundamentales (Cox et al., 2005). La primera área es "temática": la base de conocimientos se incrementa desde la institucionalidad política hacia el discernimiento de problemáticas actuales, tales como distribución del ingreso, derechos humanos, equidad, medioambiente, ciencia y tecnología. La segunda área es "cuantitativa": aumenta la presencia de la formación ciudadana en la trayectoria escolar, vale decir, se encuentra presente en todo el proceso educativo, desde su ingreso hasta su finalización. Junto con ello, los contenidos de la formación ciudadana pasan a estar distribuidos en varias asignaturas sin someterlos al arbitrio particular de una mirada (concepción maximalista).

\footnotetext{
${ }^{2}$ La asignatura ha tenido varios reveses y demoras. El año 2016, el Mineduc planteó una asignatura en educación ciudadana que iba a ser implementada durante 2017. No se sabe a ciencia cierta qué ocurrió, puesto que durante septiembre de 2017, la presidenta Michelle Bachelet, en cadena nacional, menciona que habrá una asignatura en educación ciudadana para los últimos niveles de enseñanza media ( $3^{\circ}$ y $4^{\circ}$ Medio). Si bien es cierto que han existido bastantes problemas y discusiones en materia curricular (contenidos, objetivos, propósitos, evaluación, entre otros), la información que se posee es bastante vaga al respecto. Por esta razón se hace difícil la puesta en marcha "oficial" de la asignatura para el año 2018, aunque muchos establecimientos educacionales ya la poseen como electivo.

${ }^{3}$ El Mineduc ofrece su propuesta de formación ciudadana desde un punto de vista competencial. Las propuestas del concepto de competencias en educación, y específicamente en el área de formación ciudadana, se pueden encontrar tanto en el texto de Schulz et al. (2008) como en el de Cox et al. (2005). No es menester de este trabajo profundizar en la formación ciudadana basada en las competencias, con sus consecuentes implicancias y problemas.
} 
Por último, la tercera área es "formativa": propone conocimientos y objetivos que se traducen en habilidades y actitudes.

El paso de la educación cívica a la formación ciudadana supone un cambio de paradigma que comprende ejes articuladores asociados a las áreas temática, cuantitativa y formativa.

Tabla 1.

Comparación entre Educación Cívica y Formación Ciudadana.

EDUCACIÓN CÍVICA

Foco en la institucionalidad política

\section{FORMACIÓN CIUDADANA}

Triple foco: institucionalidad política, ampliación temática a problemas actuales de la sociedad y a las competencias para resolver conflictos.

Realizada en los últimos cursos de la Presente a lo largo de la trayectoria escolar Educación Media.

Orientada a la adquisición de Orientada a la adquisición de conocimientos cuyo centro son los conocimientos, habilidades y actitudes en contenidos. ambientes y prácticas con predominio de relaciones participativas y democráticas.

Cambio de paradigma: de Educación Cívica a Formación Ciudadana (Cox et al., 2005).

El cambio de paradigma apunta hacia un enriquecimiento de los fundamentos, fines y prácticas de la educación en ciudadanía. Los conceptos y áreas temáticas, desde la teoría, son llevados a la práctica de acuerdo a las realidades en que se encuentra inmersa la escuela y el proyecto educativo que posea, es decir, se trata de situar en el contexto y en los proyectos educativos de cada escuela aquellos objetivos perseguidos por la formación ciudadana (Mineduc, 2016). 
A partir de la praxis, el currículo debiera promover una "educación para la ciudadanía", enfoque que establece como elementos centrales la adquisición por parte de las/los estudiantes de un conjunto de herramientas (el conocimiento-comprensión, habilidades y aptitudes, valores y disposiciones) que les permitan participar en forma activa y sensible en los roles y responsabilidades que tendrán en sus vidas adultas como miembros de la sociedad (Mineduc, 2014).

En este sentido, la escuela se concibe como un espacio privilegiado para el desarrollo de experiencias democráticas organizadas educativamente para incidir de manera efectiva en la sociedad (Tedesco, 2007). Por tanto, la formación ciudadana (Mineduc, 2016) integra conocimientos que permiten comprender la historia nacional, las instituciones políticas, las estructuras y los procesos del Gobierno y la vida política (educación cívica) y, a su vez, promueve que los estudiantes tengan un rol activo dentro de la comunidad educativa, aprendiendo a través de la participación la importancia que posee, desde la escuela, la experiencia y valoración de una comunidad democrática.

Este tipo de enfoque pedagógico reconoce cuatro dominios que se deberían integrar para lograr ciertas habilidades y la apropiación de la complejidad que hoy en día posee, en la teoría y en la práctica, el concepto de ciudadanía (Cox et al., 2005). En primer lugar, un dominio en "convivencia escolar democrática" que tienda a establecer relaciones sociales comprensivas de la diversidad y la tolerancia, necesarias para el desarrollo de la persona en un contexto globalizado que hace cada vez más evidente el carácter multicultural de la sociedad de la información. En segundo lugar, un dominio basado en el "enfoque de derechos humanos", en un contexto en que existen o existieron gobiernos autoritarios. Este dominio en particular debe estar orientado con especial énfasis en los derechos de las/os niñas/os. En tercer lugar, un dominio de "desarrollo de los conceptos de ciudadanía y democracia" más allá de la adquisición de conocimientos. El objetivo en este dominio es lograr integrar el entendimiento de las implicancias de estos conceptos en el marco de un aprendizaje activo y crítico de la información recibida. En cuarto lugar, un dominio referido al "aprendizaje multidisciplinario" que garantice la comprensión de los fenómenos políticos desde diversas perspectivas, contenidos, objetivos y disciplinas, fomentando una ampliación de la mirada frente a los fenómenos sociopolíticos nacionales y mundiales. 
Estos cuatro dominios se encuentran asociados a los objetivos de la Ley 20.911, pero además la Ley busca articular estos dominios y objetivos con los distintos Proyectos Educativos Institucionales (PEI), así como también con los Planes de Mejoramiento Educativo (PME). Lo anterior sin dejar de lado otras políticas nacionales que se encuentran en sintonía con la formación ciudadana, como lo es la Política de Convivencia Escolar (Mineduc, 2015) y la Ley de Inclusión (Mineduc, 2017). La formación ciudadana es una "política educativa nacional", por lo que se debe articular a otras políticas educativas. De este modo, se entiende la calidad de la educación en un sentido amplio ${ }^{4}$ (Mineduc, 2014).

Es complejo comprender este concepto desde un punto de vista de contenidos, ya sea asociado a un plan curricular, una asignatura en particular o unidades temáticas asociadas a Objetivos Fundamentales Transversales (Mineduc, 2003), convirtiendo esta articulación más bien en ambigüedad por su falta de claridad. De tal modo que el problema sustancial que plantea la política nacional en formación ciudadana será, principalmente, articular los planes de formación ciudadana, que por ley todos los establecimientos educacionales deben poseer, con la futura asignatura en formación ciudadana que será incorporada en el último ciclo de la trayectoria escolar.

Existen algunos atisbos de respuesta al respecto. Por un lado, los planes de formación ciudadana tienden a incluir acciones que enfatizan la vida en sociedad (enfoque de contexto), promoviendo la participación y valores para una sana convivencia dentro de una comunidad, para así formar un sentido de identidad y pertenencia en la comunidad educativa, como también respecto del lugar en que se encuentra inmersa dicha comunidad (PNUD, 2018). En cuanto a la comprensión de la institucionalidad política, conocimientos de economía, globalización y fenómenos de carácter mundial, rebasando lo local y potenciando la comprensión de ser ciudadanos del mundo (Cortina, 2003), tal parece ser que quedarán asociados a la asignatura de educación ciudadana.

En consecuencia, la política educativa en formación ciudadana tiene un carácter mixto, vale decir, incluye una asignatura particular (nivel curricular), en tanto que la

\footnotetext{
${ }^{4}$ Existen dificultades para definir lo que se entiende por calidad en educación. Hablar de calidad en la educación en un sentido amplio es más bien una premisa que una definición a seguir en tanto política educativa. La literatura especializada sigue entendiendo el concepto de calidad asociado a niveles de logro o rendimiento académico (Casassus, 2014). En este sentido cabe preguntarse ¿qué se quiere medir o lograr en formación ciudadana (y si acaso es posible aquello)?
} 
escuela debe poseer un plan con acciones que promuevan la formación ciudadana dentro de su proyecto educativo (nivel institucional). La concepción mixta en formación ciudadana (ACE, 2016) es concomitante con el enfoque mundial de educación en ciudadanía a través de evaluaciones estandarizadas internacionales (ICCS) que miden competencias en dimensiones institucionales y cívicas (dimensión política), junto con una mirada más cultural y activa en el ejercicio cotidiano en ciudadanía (dimensión social).

El Mineduc promueve una educación en ciudadanía que es concomitante con las políticas que en materia educativa son difundidas por instituciones globales como el Banco Mundial y la Organización para la Cooperación y el Desarrollo Económicos (OCDE/Banco Mundial, 2009) y la Organización de las Naciones Unidas para la Educación, la Ciencia y la Cultura (INNOVEMOS, 2008); como también en el contexto local americano por instituciones como la Organización de Estados Americanos y el Banco Interamericano de Desarrollo (Espínola, 2005). Además, este último organismo ha sido promotor de los seminarios entre los Ministros de Educación de América, asumiendo en su agenda el compromiso con la educación de la ciudadanía y la democracia, al señalar que:

No es posible concebir a los alumnos como futuros ciudadanos activos si su experiencia de aprendizaje de la ciudadanía ha sido predominantemente pasiva. La investigación comparada —así como esfuerzos nacionales recientes de deliberación y acuerdo sobre educación para la ciudadanía activa - convergen sobre la necesidad de un abordaje pedagógico que combine el aprendizaje a través de la enseñanza formal de contenidos, la discusión de asuntos sociales y políticos, y la reflexión sobre la experiencia. (Cox et al., 2005, p. 23)

\section{METODOLOGÍA DE TRABAJO}

El trabajo realizado en la asesoría surge a la luz de un convenio de colaboración entre la Universidad Católica Silva Henríquez y el Mineduc, cuyo propósito es orientar la elaboración, construcción, implementación y evaluación de Planes de Formación Ciudadana en el contexto de los objetivos de la Ley 20.911 a establecimientos educacionales municipales durante los años 2016 a 2018. Este programa de asesoramiento 
tuvo dos cohortes: años 2016-2017 y años 2017-2018. La labor de la asesoría contempló desarrollar estrategias de trabajo con tres Departamentos Provinciales de Educación de la Región Metropolitana, a saber: Deprov. Oriente, Deprov. Cordillera y Deprov. Sur. Cada Departamento Provincial, en conjunto con los asesores, generó instancias de acción de acuerdo a los diversos contextos.

En el caso particular del Deprov. Sur, el universo de establecimientos educacionales fue de un total de 31 colegios municipales repartidos en distintas comunas de la zona sur, tales como El Bosque, La Cisterna, Lo Espejo, La Granja, San Ramón, San Bernardo, Calera de Tango, Buin y Paine. Los establecimientos educacionales fueron distribuidos en dos cohortes. La primera cohorte (2016-2017) contempló veinte establecimientos educacionales, en tanto que la segunda cohorte (2017-2018) contempló once.

La metodología de trabajo utilizada ha sido mixta con una preponderancia cualitativa (Hernández, Fernández, \& Baptista, 2010), abarcando estrategias de trabajo para el Departamento Provincial Sur y las distintas comunidades educativas para así identificar las percepciones que se tienen en torno a la formación ciudadana, además de sensibilizar sobre los objetivos de la Ley.

Las estrategias de trabajo se fueron desarrollando desde un sentido amplio (Deprov. Sur y establecimientos educacionales) hacia un trabajo más específico (talleres y visitas a terreno a los distintos establecimientos educacionales) orientando, en este último punto en particular, un diseño metodológico basado en la investigación-acción (Hernández et al., 2010), debido a que el objetivo de la asesoría consistió en elaborar, organizar y operacionalizar diversas actividades que permitieran a los distintos establecimientos construir sus propios planes de formación ciudadana de acuerdo a las exigencias que la Ley 20.911 dispone. A continuación, se presentan las estrategias de trabajo y las actividades que se llevaron a cabo.

\section{JORNADAS MASIVAS}

El propósito de esta actividad fue difundir la política nacional educativa en formación ciudadana. El objetivo planteado consistió en dar a conocer los objetivos y fines de la Ley 
20.911 y sensibilizar en torno a ellos, a la vez que identificar las diversas percepciones que poseen los distintos establecimientos sobre el significado y sentido de la formación ciudadana en las escuelas. Para ello, se generaron instancias de diálogo con los encargados de formación ciudadana de los establecimientos educacionales para visibilizar las representaciones acerca de la democracia y la ciudadanía, y las prácticas que, en consecuencia, son llevadas a cabo en las escuelas. Estos elementos permitieron detectar las primeras concepciones de lo que es la formación ciudadana, como también discutir sobre las dificultades de su implementación en el sistema escolar. Por tanto, se buscó que los establecimientos educacionales reflexionaran sobre los propósitos de la Ley y las dificultades que esta tendría en su implementación (nudos críticos).

\section{TALLERES}

El propósito de los talleres consistió en generar un trabajo más focalizado a partir del diagnóstico que los asesores elaboraron acerca de los nudos críticos al interior de los establecimientos educacionales. El diagnóstico a trabajar es específico, pues cada Departamento Provincial posee características particulares. El trabajo de diagnóstico permite desarrollar reflexivamente la posibilidad que los encargados de los planes de formación ciudadana y la propia comunidad educativa dialoguen acerca de cuáles son las principales tensiones y dificultades a la hora de implementar un plan de formación ciudadana. Las principales respuestas se explicitaron en tres criterios:

a) Nudos críticos en los actores del establecimiento educacional.

b) Nudos críticos en los documentos institucionales del establecimiento educacional.

c) Nudos críticos en el currículum escolar.

\section{INSTRUMENTOS}

La discusión posterior de los talleres expuesta por los asesores y socializada en plenario con los encargados del plan de formación ciudadana implicó un desarrollo práctico de trabajo en base a instrumentos elaborados específicamente para los nudos críticos de los 
establecimientos educacionales del Deprov. Sur. El objetivo principal de los distintos instrumentos es que los encargados puedan desarrollar y articular objetivos dentro del Proyecto Educativo Institucional (PEI) en clave ciudadana para, en un segundo momento, desarrollar acciones que permitan cumplir dichos objetivos y, finalmente, evaluar las acciones implementadas en virtud de su grado de significación y sentido dentro de la comunidad educativa. Todo esto sin olvidar que las acciones también deben tributar a los objetivos planteados por la Ley 20.911.

Los resultados de los instrumentos fueron socializados con los distintos establecimientos educacionales. El objetivo del trabajo consistió en desarrollar tanto la fundamentación como las acciones del plan de formación ciudadana. Este trabajo permitió tener el primer borrador del plan de todos los establecimientos educacionales del Deprov. Sur adscritos al convenio.

Por su parte, los asesores leyeron los borradores de los planes y enviaron sugerencias y observaciones pertinentes para cada caso particular. Una vez devuelto el plan de formación ciudadana con las sugerencias y observaciones trabajadas, se obtuvo el plan final que se presentará en el primer Consejo Escolar del año siguiente para su respectiva validación y aprobación.

\section{VISITAS A ESTABLECIMIENTOS EDUCACIONALES}

El propósito principal de las visitas a terreno fue forjar un tipo de trabajo más estrecho y vinculante con los distintos contextos educativos, acompañando la implementación del plan de formación ciudadana y analizando de manera particular las dificultades que cada establecimiento educacional pudiera tener en la operacionalización del plan.

Este trabajo ayudó a dilucidar interrogantes de carácter más práctico tanto en la planificación como implementación de acciones con sentido dentro las escuelas. Además permitió conocer a los distintos actores de la comunidad educativa, promoviendo formas de comunicación directa con los encargados del plan. Hizo posible también que los asesores conocieran en terreno los distintos establecimientos educacionales. 
Todas estas estrategias antes mencionadas permitieron a los asesores vislumbrar cuáles eran los principales elementos que deberían reforzarse en cuanto a los propósitos y objetivos de la Ley 20.911, para así poder elaborar y concretar planes de formación ciudadana que permitieran a las distintas escuelas asociadas al convenio del Deprov. Sur generar acciones cuyos objetivos, alcances y fines fueran pertinentes para la comunidad educativa.

En este sentido, se buscó articular aquellos elementos sello que hablen desde las propias comunidades cuya finalidad sea que las actividades tengan un sentido particular y situado (Apple \& Beane, 1999). Por esta razón se hizo principal hincapié en que los planes de formación ciudadana debían vincularse con el PEI, estableciendo la necesidad de que no solamente cada acción creada tribute a los objetivos de la Ley, cosa por lo demás importante y necesaria, sino que, por sobre todo, que el plan de formación ciudadana emerja a partir del PEI, y de esta manera las acciones se encuentren tanto articuladas con los objetivos de la Ley como con los objetivos sello que el propio establecimiento educativo crea pertinente.

\section{CONCLUSIONES}

Los resultados de la experiencia avalan que los establecimientos educacionales se encuentran trabajando de una manera más consciente y articulada en los planes de formación ciudadana. La elaboración del plan posee fundamentos y comienza a gestarse una intencionalidad acorde a los distintos proyectos educativos. Sus objetivos y fines comienzan a ser cada vez más claros al encontrarse articulados con los proyectos educativos, lo que les da un sello propio.

Los planes de formación ciudadana de los establecimientos educacionales, con sus respectivas acciones, brindan una particular importancia al sentido de pertenencia, por lo que las actividades de participación comunitaria, la difusión del PEI y las actividades que promueven la identidad con la escuela pasan a ser elementos fundamentales. De esta manera, las acciones están enfocadas a la vida en común sobre la base de una relación sana con quien se convive cotidianamente, entendiendo la formación ciudadana como un espacio 
de encuentro y relación con los cercanos, tal y como señala el Estudio sobre la puesta en marcha del Plan de Formación Ciudadana (PNUD, 2018). Esta dimensión trabajada por los planes de formación ciudadana de los establecimientos educacionales, no obstante, deja en un ámbito secundario la dimensión cívica y política.

Así también, gran parte de las escuelas han podido generar procesos de articulación entre los objetivos de la Ley y los objetivos del PEI que se han traducido en actividades sello. A modo de ejemplo, una escuela en la comuna de El Bosque tiene un fuerte sello en derechos humanos; escuelas en las comunas de San Bernardo, Calera de Tango y Paine, en el área del medio ambiente y la ecología; escuelas en las comunas de San Bernardo, La Granja y San Ramón, en el área de diversidad cultural. Lo interesante de estos establecimientos educacionales es que han podido interpretar adecuadamente el contexto donde se encuentran situados y las problemáticas propias que van emergiendo.

A pesar de lo anterior, la experiencia en la asesoría sugiere aún la existencia de tensiones dentro de la propia comunidad. El taller correspondiente a visibilizar nudos críticos al interior de la escuela permite explicitar la ausencia persistente de trabajo colaborativo y colegiado. Más aún, permanecen aquellas actividades con un fuerte componente disciplinar asociado a los objetivos de aprendizaje curriculares de las asignaturas de Filosofía e Historia y Geografía, en tanto que el profesorado de otras asignaturas encuentra que no es propio de su disciplina la formación ciudadana. Es interesante este punto pues, para citar un ejemplo, el análisis de la realidad nacional, la deliberación y el debate, y la lectura de noticias se entiende como ajeno a la educación disciplinar de las asignaturas. Esta concepción de la formación ciudadana instala aún la premisa de que los niveles de dominio (conocimiento, habilidades y actitudes) siguen siendo propios de la asignatura de Historia y no de la formación integral del estudiantado, lo que favorece que se generen actividades curriculares dentro del plan de formación ciudadana que no tienen mayor vinculación y sintonía con los objetivos sello que el establecimiento educacional propone.

En virtud de aquello, considerando el trabajo realizado en la asesoría y el análisis que emerge de los distintos instrumentos implementados se definen los siguientes criterios 
que proponen desafíos para seguir avanzando en el desarrollo de la política nacional en formación ciudadana:

1. Articular el plan de formación ciudadana a los documentos institucionales de la comunidad educativa más allá del Proyecto Educativo Institucional (PEI), con especial énfasis en la vinculación que debiere tener con el Plan de Mejoramiento Educativo (PME).

2. Articular el plan de formación ciudadana con otras políticas nacionales educativas, como lo es la Política en Convivencia Escolar y Ley de Inclusión. Estas políticas nacionales son importantes, pues entienden a la educación como un espacio de formación integral (Mineduc, 2016; 2017).

3. Es importante que todas las acciones del plan de formación ciudadana se encuentren fundamentadas en los propósitos y fines que propicie la escuela junto con los objetivos sello emanados del propio establecimiento educativo.

4. Difundir, socializar y retroalimentar el plan de formación ciudadana y sus acciones con los distintos actores de la comunidad educativa. Esta tarea debiese ser un desafío permanente en el tiempo.

5. Tener en cuenta que el plan de formación ciudadana brinda la posibilidad de coordinar todos los proyectos al interior del establecimiento educativo, así como actividades, contenidos y objetivos de las distintas asignaturas. De esta manera, permite un trabajo multidisciplinario con un enfoque en los aprendizajes significativos no sólo de los estudiantes, sino de los distintos actores que participan de la comunidad, favoreciendo una cultura escolar con sentido.

6. Considerar la importancia de la participación y reflexión que puede surgir a partir de los estudiantes debido a que son los actores principales dentro del proceso de su propia formación en ciudadanía. Tal y como señala el Mineduc (2016): "la ciudadanía no puede ser un discurso, sino una realidad representada en una práctica efectiva" (p.18).

Finalmente, uno de los desafíos que señala el trabajo de la asesoría para las escuelas es pensar reflexivamente qué implica formar ciudadanos críticos, entendiendo por ello la posibilidad de expresión de las distintas voces que se pueda promover tanto a nivel institucional (escuela) como a nivel curricular (aula) (Apple \& Beane, 1999). Por otra parte, 
resaltar la importancia que tiene el contexto donde se encuentra la comunidad educativa, y el grado de reflexión y participación que de esta pueda emerger. Así también la mirada que se posea de la sociedad, y la importancia de la contingencia y la realidad nacional en tanto espacios de deliberación política en el aula.

\section{REFERENCIAS}

ACE. (2016). Formación ciudadana en el sistema escolar chileno: una mirada a las prácticas actuales y recomendaciones de mejora. Santiago: Agencia de Calidad de la Educación.

Apple, M., \& Beane, J. (1999). Escuelas democráticas. Madrid: Morata.

Casassus, J. (2014). La escuela y la (des)igualdad. Santiago: LOM.

Cerda, A., Egaña, L., Magendzo, A., Santa Cruz, E., y Varas, R. (2004). El complejo camino de la formación ciudadana. Santiago: LOM.

Comisión Formación Ciudadana (2004). Informe Comisión Formación Ciudadana. Santiago, Chile. Recuperado de https://es.slideshare.net/annyhen/informe-formacinciudadana-en-chile

Comisión Nacional para la Modernización de la Educación (1994). Informe. Los desafíos de la educación chilena frente al siglo 21. Santiago: Editorial Universitaria.

Consejo Asesor Presidencial contra los Conflictos de Interés, el Tráfico de Influencias y la Corrupción (2015). Informe final. Santiago. Recuperado de http://consejoanticorrupcion.cl/informe/

Cortina, A. (2003). Ciudadanos del mundo. Hacia una teoría de la ciudadanía. Madrid: Alianza. 
Cox, C., Jaramillo, R., y Reimers, F. (2005). Educar para la ciudadanía y la democracia para las Américas: una agenda para la acción. Nueva York: Banco Interamericano de Desarrollo.

Espínola, V. (Ed.). (2005). Educación para la ciudadanía en un mundo globalizado. Washington, D. C.: Banco Interamericano de Desarrollo.

Hernández, R., Fernández, C., y Baptista, P. (2010). Metodología de la Investigación. México D. F.: McGraw-Hill.

Innovemos. (2008). Convivencia democrática, inclusión y cultura de paz. Lecciones desde la práctica educativa en América Latina. Santigo: UNESCO.

Lechner, N. (1988). Los patios interiores de la democracia. Subjetividad y política. Santiago: FLACSO.

Lechner, N. (1987). La democratización en el contexto de una cultura posmoderna. En N. Lechner, Cultura política y democratización (págs. 253-262). Santiago: FLACSO, CLACSO, ICI.

Ley N ${ }^{\circ}$ 20.911. Diario oficial de la República de Chile, Santiago, Chile, 2 de abril de 2016.

Mineduc (2003). Educación cívica y ejercicio de la ciudadanía. Los estudiantes chilenos en el Estudio Internacional de Educación Cívica. Santiago: Mineduc.

Mineduc (2013). Orientaciones técnicas y guiones didácticos para fortalecer la formación ciudadana. $7^{\circ}$ Básico a $4^{\circ}$ Medio. Santiago: Mineduc.

Mineduc. (2014). Orientaciones e instrumentos de evaluación diagnóstica, intermedia y final en formación ciudadana. Santiago: Mineduc.

Mineduc. (2015). Política Nacional de Convivencia Escolar 2015/2018. Santiago: Editor.

Mineduc. (2016). Orientaciones para la elaboración de un plan de formación ciudadana. Santiago: Mineduc. 
Mineduc. (2017). Ley de inclusión escolar. Santiago: Mineduc.

OCDE/Banco Mundial. (2009). La educación superior en Chile. Santiago: Mineduc.

PNUD. (2002). Nosotros los chilenos. Un desafío cultural. Santiago: PNUD.

PNUD. (2004). El poder: ¿para qué y para quién? Santiago: PNUD.

PNUD. (2014). Auditoría a la democracia. Más y mejor democracia para un Chile inclusivo. Santiago: PNUD.

PNUD. (2015). Los tiempos de la politización. Santiago: PNUD.

PNUD. (2017). Desiguales. Orígenes, cambios y desafíos en la brecha social en Chile. Santiago: PNUD.

PNUD. (2018). Estudio sobre la puesta en marcha del plan de formación ciudadana. Santiago: PNUD.

Schulz, W., Fraillon, J., Ainley, J., Losito, B., y Kerr, D. (2008). Estudio Internacional sobre Educación Cívica y Ciudadana. Amsterdam: Agencia Internacional para la Evaluación y Rendimiento Educativo.

Tedesco, J. C. (2007). Educar en la sociedad del conocimiento. Buenos Aires: FCE.

Touraine, A. (2015). ¿Qué es la democracia? México D. F.: FCE. 